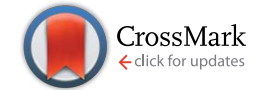

Cite this: RSC Adv., 2016, 6, 5999

\title{
3-Dimensional porous nanocomposite scaffolds based on cellulose nanofibers for cartilage tissue engineering: tailoring of porosity and mechanical performance $\uparrow$
}

\author{
Narges Naseri, ${ }^{a}$ Jean-Michel Poirier, ${ }^{a}$ Lenart Girandon, ${ }^{\mathrm{b}}$ Mirjam Fröhlich, ${ }^{\mathrm{b}}$ \\ Kristiina Oksman ${ }^{\mathrm{a}}$ and Aji P. Mathew ${ }^{\star a c}$
}

Fully bio-based 3-dimensional porous scaffolds based on freeze-dried cellulose nanofibers (70-90 wt\%) stabilized using a genipin crosslinked matrix of gelatin and chitosan were prepared. Morphology studies using scanning electron microscopy showed that the scaffolds have interconnected pores with average pore diameters of 75-200 $\mu \mathrm{m}$ and nanoscaled pore wall roughness, both favorable for cell interactions with cartilage repair. X-ray tomography confirmed the 3-dimensional homogeneity and interconnectivity of the pores as well as the fibrillar structure of the scaffolds. The compression modulus of the scaffolds (1-3 $\mathrm{MPa})$ at room conditions was higher than natural cartilage $(\approx 1 \mathrm{MPa})$. The lowered compression modulus of $10-60 \mathrm{kPa}$ in phosphate buffered saline (PBS) at $37{ }^{\circ} \mathrm{C}$ was considered favorable for chondrogenesis. The current study therefore successfully addressed the challenge of tailoring the pore structure and mechanical properties simultaneously for cartilage regeneration. Furthermore, the scaffolds' high porosity ( $\approx 95 \%$ ), high PBS uptake and good cytocompatibility towards chondrocytes are considered beneficial for cell attachment and extracellular matrix (ECM) production.

Received 20th December 2015 Accepted 5th January 2016

DOI: $10.1039 / c 5 r a 27246 g$

www.rsc.org/advances
In cartilage tissue engineering, scaffolds are expected to imitate the functions of damaged cartilage and provide a 3dimensional (3D) environment for cell growth and ECM production. $^{3,4}$ Typically, the tailoring of scaffold porosity with pore sizes in the range of $200-300 \mu \mathrm{m}$ is considered a benchmark for cartilage regeneration. ${ }^{4,13}$ Such porous scaffolds can be prepared using many different processes, such as freeze-drying, $\mathrm{CO}_{2}$ foaming, electrospinning or cryogelation, with a wide variety of polymers as found in literature., ,14-16 $^{-1}$

Chitosan and gelatin based scaffolds have been reported to be beneficial for soft load bearing tissues. Positive charges on the surface of chitosan can promote chondrocyte growth thus beneficial for cartilage repair., ${ }^{3,4}$ Gelatin is partially derived from collagen, which is the main protein component of connective tissues as cartilage, skin and bone. Furthermore, a blend of these polymers absorbs water and forms a hydrogel whereby allowing fluid to be retained in the scaffold structure leading to a higher compression modulus similar to natural soft tissue. ${ }^{4,13,17,18}$ A recent study on chitosan/gelatin blends with random and aligned pore structures prepared with a freezedrying process showed a compression modulus in the range of $5 \mathrm{kPa}$ (for randomly aligned scaffolds) to $30 \mathrm{kPa}$ (in the vertical direction of the aligned scaffold), when tested at $37{ }^{\circ} \mathrm{C}$ after conditioning in PBS medium. ${ }^{18}$

To enhance the mechanical performance of biopolymers and their blends, different types of reinforcements obtained from
${ }^{a}$ Division of Materials Science, Luleå University of Technology, 97187 Luleå, Sweden. E-mail: aji.mathew@ltu.se; aji.mathew@mmk.su.se; Fax: +46 92049 1399; Tel: +46 920492024

${ }^{b}$ Educell ltd., Prevale 9, 1236 Trzin, Slovenia

${ }^{c}$ Division of Materials and Environmental Chemistry, Stockholm University, SE-10691 Stockholm, Sweden

$\dagger$ Electronic supplementary information (ESI) available. See DOI: 10.1039/c5ra27246g 
natural materials have been used; the most important examples being derivatives of cellulose and chitin.,.$^{\mathbf{8 , 9 , 1 4 , 1 5}}$ Our earlier studies have also demonstrated that solution cast fibrous nanocomposite structures with high cellulose nanofibers concentrations (75 wt\%) and collagen provide mechanical performance suitable for ligaments. ${ }^{89}$ Also, our previous studies have demonstrated the noncytotoxicity of nanocellulose from different sources and their potential in medical applications..$^{15,19,20}$

In the current study, 3D nanocomposite scaffolds for cartilage regeneration were processed via freeze-drying technique, where porous cellulose nanofiber structures were bound together and mechanically/dimensionally stabilized using low amounts of crosslinked chitosan/gelatin blend system. The pore structure developed during the processing is expected to impact the moisture uptake and mechanical performance of the resultant scaffolds. Moreover, the pore structure and sizes, which favor the movement of fluids through the scaffold, creating drag forces, ${ }^{21}$ as well as the development of ECM, is known to contribute to the load bearing under in vivo conditions. Though tailoring of mechanical properties using nanocellulose have received some attention in tissue engineering, ${ }^{\mathbf{8 , 9 , 2}}$ limited studies are available on tailoring the pore structure in bionanocomposites and understanding the effect of porosity on the mechanical performance. It is highly challenging to tailor the pore structure and mechanical properties required for tissue regeneration, simultaneously, as increase in porosity generally decreases the mechanical properties and vice versa. The pore structure and porosity of the scaffold was tailored in the current study by varying the suspension concentrations, fibers/matrix ratio and crosslinking with the aim to obtain optimal mechanical properties and chondrocyte attachment and proliferation to obtain extracellular matrix of optimal quality. It was expected that these $3 \mathrm{D}$ porous structures could act as templates for the formation of new tissue and act as guidance for cell growth while facilitating nutrient and oxygen transport.

The structural morphology of the produced scaffolds was observed by scanning electron microscopy (SEM) and X-ray tomography. Mechanical properties in room condition and in PBS medium, moisture uptake, density and porosity, as well as in vitro biodegradation and cytocompatibility towards chondrocytes were also investigated.

\section{Experimental}

\subsection{Materials}

High-purity cellulose from softwood fibers (Norwegian spruce) with high cellulose content (95\% cellulose, $4.5 \%$ hemicellulose and $0.1 \%$ lignin content as provided by Domsjö Fabriker AB, Sweden) was used as starting material for the production of cellulose nanofibers. Medium $M_{\mathrm{w}}$ chitosan (DD $\approx 75-85 \%$ ), acetic acid, gelatin, as well as phosphate buffered saline (PBS) and genipin were purchased from Sigma-Aldrich, Germany.

\subsection{Methods}

Processing of cellulose nanofibers. Cellulose fibers were dispersed in distilled water at a concentration of $2 \mathrm{wt} \%$ using a mechanical blender, Silverson L4RT (England), at $6000 \mathrm{rpm}$ for $15 \mathrm{~min}$. Then, the suspension was ground using an ultra-fine grinder, MKCA 6-3 from Masuko (Tokyo, Japan) to obtain nanofibers (CNF), following the procedure reported by Mathew et al. ${ }^{9}$ As the pore structure required for the cartilage application is in micron scale range,,$^{\mathbf{4 1 3 , 2 3 , 2 4}}$ the fibrillation process was aimed towards obtaining relatively coarser fibrils as they are expected to give larger pore sizes for the scaffold compared to their finer counterparts.

The atomic force microscopy of the prepared nanofibers (Fig. 1a) showed nanosized fibers with diameters in the range of 19-38 nm (Fig. 1b), based on the measurements using the Nanoscope V software (Santa Barbara, CA, USA). The diameter of nanofibers was measured from the height to compensate the tip broadening effect. The photograph of the gels of CNF obtained after grinding is also shown (Fig. 1c).

The suspension was centrifuged for $30 \mathrm{~min}$ and the rotor speed was $1500 \mathrm{rpm}$ to remove water and obtain highly concentrated gel, typically between 7 and $10 \mathrm{wt} \%$, which was used for scaffold processing.

Processing of the nanocomposite scaffolds. The matrix (gelatin/chitosan), the nanofibers and the genipin were mixed in a one step process and crosslinked in situ. The gelatin

c)
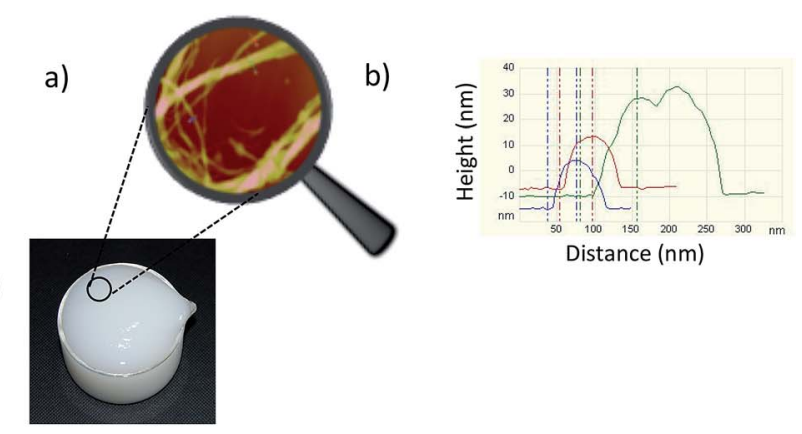

d)

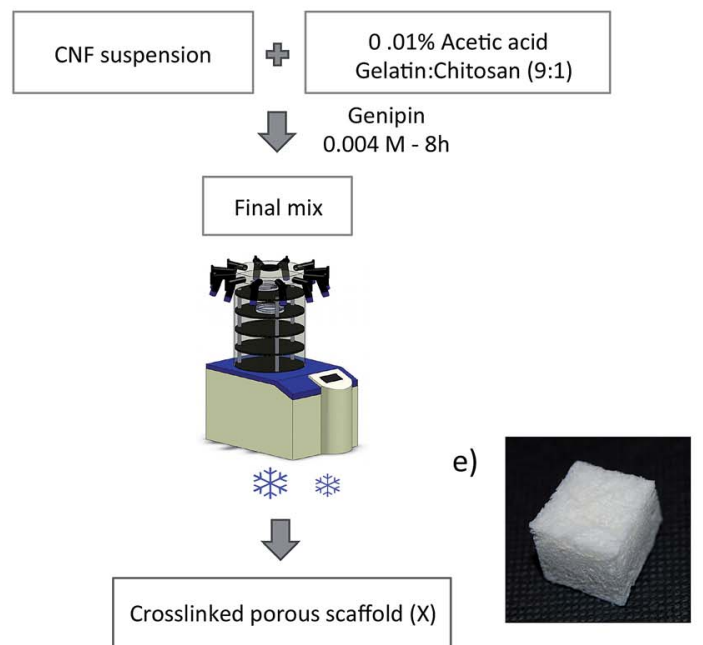

Fig. 1 (a) Atomic force microscopy height image of cellulose nanofibers from high purity cellulose; (b) diameter determination based on the height; (c) the photograph of CNF gel; (d) the whole processing route for the preparation of porous scaffold, and (e) the photograph of the produced scaffold. 
(G)/chitosan (Ch) mixture in a ratio of $(9: 1)$ was dissolved in $0.01 \%$ acetic acid medium containing $0.004 \mathrm{M}$ genipin solution. Gelatin/chitosan mixture in the ratio of $9: 1$ is referred to as the matrix $(M)$ throughout the manuscript. The nanofiber suspensions were mixed with this solution in appropriate amounts to obtain final nanocomposites with different compositions. All samples were placed in plastic Petri dishes, frozen at $-30{ }^{\circ} \mathrm{C}$ and freeze-dried at $-70{ }^{\circ} \mathrm{C}$ in a vacuum (0.0026 mbar). The complete process with a photograph of the produced scaffold is shown in Fig. 1d and e, respectively. All scaffolds prepared in this work and their compositions are listed in Table 1.

\subsection{Characterization}

Scanning electron microscopy (SEM). The samples were cryogenically fractured to preserve the structure and SEM micrographs were acquired with a SEM JEOL JSM-6460LV at voltages of 5 and $15 \mathrm{kV}$. All samples were sputter-coated with gold for $50 \mathrm{~s}$ at $50 \mathrm{~mA}$ to avoid electron charging. The pore sizes were measured from the SEM images using SemAfore.

For high-resolution images, MAGELLAN 400 XHR-SEM (FEI Company, Eindhoven, The Netherlands) was used. The samples were placed on carbon tape, coated with tungsten, and observed under the SEM at an acceleration voltage of $3 \mathrm{kV}$.

X-ray tomography. 3D structure of pores was reconstructed using a Zeiss XRadia XRM 520 X-ray tomograph. For these images, an X-ray tube voltage of $40 \mathrm{keV}$ was used, which results in a broad spectrum of X-ray energies, with a maximum of about $40 \mathrm{keV}$. No filter was used on the source. The beam produced by the source is a cone-beam, which provides a geometrical magnification of the image depending on the source-detector distance and the position of the sample between the two. In this case the sample was placed at $9 \mathrm{~mm}$ from the source and $9 \mathrm{~mm}$ from the detector. 1601 radiographs were acquired over $360^{\circ}$ with an exposure time of $2 \mathrm{~s}$ and $12 \mathrm{~s}$ per projection, respectively for the $4 \times$ and $20 \times$ images. The tomographic reconstruction was performed using the Zeiss reconstructor software with a correction for the center of rotation.

Density and porosity. The density was calculated by cutting approximately cubic samples, measuring all dimensions, weighing them and dividing the weight by the volume. Each measurement was taken three times and the results reported

Table 1 Composition, density, porosity and average pore size of the processed samples $^{a}$

Density Porosity Average pore

Sample CNF (g) $M(\mathrm{~g})$ Sample code $\left(\mathrm{g} \mathrm{cm}^{-3}\right)(\%) \quad$ size $(\mu \mathrm{m})$

\begin{tabular}{|c|c|c|c|c|c|c|}
\hline 1 & 4 & 0.0 & $\mathrm{CNF}_{4}$ & 0.069 & 95.5 & - \\
\hline 2 & 4 & 0.4 & $\mathrm{CNF}_{4}-\mathrm{M}_{0.4}$ & 0.062 & 95.6 & $153 \pm 53$ \\
\hline 3 & 4 & 0.4 & $\mathrm{X}-\mathrm{CNF}_{4}-\mathrm{M}_{0.4}$ & 0.066 & 95.3 & $68 \pm 49$ \\
\hline 4 & 4 & 1.1 & $\mathrm{X}-\mathrm{CNF}_{4}-\mathrm{M}_{1.1}$ & 0.061 & 95.1 & $58 \pm 35$ \\
\hline 5 & 4 & 2.2 & $\mathrm{X}-\mathrm{CNF}_{4}-\mathrm{M}_{2.2}$ & 0.065 & 94.2 & $59 \pm 18$ \\
\hline 6 & 5 & 0.5 & $\mathrm{X}-\mathrm{CNF}_{5}-\mathrm{M}_{0.5}$ & 0.076 & 94.6 & $90 \pm 71$ \\
\hline 7 & 6 & 0.6 & $\mathrm{X}-\mathrm{CNF}_{6}-\mathrm{M}_{0.6}$ & 0.093 & 93.4 & $65 \pm 51$ \\
\hline
\end{tabular}

${ }^{a} M$ : matrix composed of gelatin/chitosan $(9: 1)$, X: crosslinked. are based on the average values. Porosity of the scaffolds was evaluated based on the weight and density of the scaffolds. The porosity was defined as the volume fraction of the voids $\left(V_{\mathrm{v}}\right)$ and was calculated using the following equation. ${ }^{22,23}$

$$
v=1-\frac{\rho_{\mathrm{e}}}{\rho_{\mathrm{t}}}
$$

1 where $\rho_{\mathrm{e}}$ is the experimental density of the scaffold and $\rho_{\mathrm{t}}$ is the theoretical density of a non-porous scaffold. The densities of $\mathrm{CNF}$, gelatin and chitosan were taken as 1.54, 0.98 and $0.235 \mathrm{~g}$ $\mathrm{cm}^{-3}$, respectively.

Nanoscaled pores were measured using a Micromeritics ASAP 2000 instrument and the average pore diameters were determined from nitrogen adsorption measurements at $77 \mathrm{~K}$ using the BET method. The measurements were performed after degassing the samples at $100{ }^{\circ} \mathrm{C}$ for $48 \mathrm{~h}$ in dry $\mathrm{N}_{2}$ flow.

Moisture uptake. The moisture uptake measurements were performed in PBS medium. The samples were dried overnight in a vacuum oven at $80{ }^{\circ} \mathrm{C}$, immediately weighed $\left(W_{\mathrm{d}}\right)$ and thereafter immersed in PBS. Weights were taken at different time intervals $(t)$, i.e. $30 \mathrm{~s}$ after immersion and another one 3 days later $\left(W_{t}\right)$. Every time the excess water was removed by gently tapping the samples on a dry soft tissue paper. The moisture uptake was calculated according to the following equation.

$$
\text { Moisture uptake }(\%)=\left[\left(W_{t}-W_{\mathrm{d}}\right) / W_{\mathrm{d}}\right] \times 100
$$

In order to assess the swelling of the matrix phase, samples were dried in the vacuum oven and weighed as described above and placed in a 95\% moisture desiccator. The samples were weighed every two days for two weeks in order to follow the weight gain until equilibrium. The moisture uptake was calculated using the same equation as above.

Compression tests. All compression tests were performed at $37{ }^{\circ} \mathrm{C}$ using a TA Instruments DMA Q-800 (New Castle, DE, USA), according to an adapted version of the D11621-94 standard test method. The samples were cut into square pieces with sides between 5 and $10 \mathrm{~mm}$. The thickness was about $10 \mathrm{~mm}$ for dry tests and the displacement rate was $100 \mu \mathrm{m} \mathrm{min}{ }^{-1}$ with the contact force of $0.05 \mathrm{~N}$. The compression moduli were calculated as the slope of the stress-strain curve in the linear region, below $15 \%$ strain.

For tests in PBS, the sample thickness was about $5 \mathrm{~mm}$ and the cut samples were immersed in PBS for $24 \mathrm{~h}$ before testing. The displacement rate for moduli calculation was $400 \mu \mathrm{m}$ $\min ^{-1}$ and the initial contact force was $0.02 \mathrm{~N}$, slightly adapted according to the specific rigidity of each type of sample (up to $0.05 \mathrm{~N}$ ). Compression tests were also performed in PBS at varying strain rates ranging from $100 \mu \mathrm{m} \mathrm{m^{-1 }}$ to $400 \mu \mathrm{m}$ $\min ^{-1}$ to evaluate viscoelasticity. Each test was performed at least five times and the average values were reported.

In vitro biodegradation. The biodegradation of scaffolds was investigated using conventional technique. In this method, the dried specimens were immersed in PBS under $\mathrm{pH} 7.4$ and stored in a thermostatically shaking water bath at $37^{\circ} \mathrm{C}$ for up to 28 days. The samples were removed at different times. The 
$\mathrm{pH}$ of the PBS solution was measured by $\mathrm{pH}$ meter and then replaced with fresh PBS each time. After removal of samples, the surface of the specimens was gently blotted by soft tissue paper in order to remove water. The specimens were then completely dried in a vacuum oven at $50{ }^{\circ} \mathrm{C}$ until a constant weight was achieved, and then they were weighed. The weight loss was calculated using the following equation.

$$
\text { Weight loss }(\%)=\left[\left(W_{0}-W_{t}\right) / W_{0}\right] \times 100
$$

$W_{0}$ is the sample's original weight, and $W_{t}$ is the weight of the specimen at time $(t)$.

\section{Cytocompatibility studies}

Cytocompatibility of the CNF. CNF films were fixed to cell culture dishes and the cells (adipose derived stem cells (ASCs) and L929 cell line) were seeded evenly throughout the cell culture dish. The impact of the biomaterial on cell growth and morphology was monitored and documented with photographs.

Cytocompatibility of the scaffolds. Cytocompatibility of the scaffolds was monitored in a direct contact testing system according to ISO 10993. The biomaterials were fixed in cell culture vessels and cells, namely chondrocytes, were seeded on the biomaterial, $(0.5 \times 106$ cells per $\mathrm{ml})$ in cell culture media supplemented with 10\% FBS (Fetal Bovine Serum). The scaffolds with cells were incubated at $37{ }^{\circ} \mathrm{C}$ in $5 \% \mathrm{CO}_{2}$ for 7 days. After 7 days the biomaterials were stained with MTT (3-(4,5dimethylthiazolyl-2)-2,5-diphenyltetrazolium bromide) and inspected for the presence of live cells on the upper surface of the scaffolds. Biomaterial with no seeded cells was regarded as a negative control.

\section{Results and discussion}

\subsection{Morphology and pore structure of scaffolds}

The SEM images were evaluated to understand the effect of crosslinking and suspension composition on scaffold morphology. $\mathrm{CNF}_{4}-\mathrm{M}_{0.4}$ and $\mathrm{X}-\mathrm{CNF}_{4}-\mathrm{M}_{0.4}$ (Fig. 2a and b) showed the pore sizes in the micrometer range in both cases. Uncrosslinked scaffolds had a wide distribution in pore size (Fig. 2a) while crosslinking slightly enhanced the overall homogeneity of the structure and decreased the pore size (Fig. 2b).

The morphologies of X-CNF $4-\mathrm{M}_{0.4}, \mathrm{X}-\mathrm{CNF}_{4}-\mathrm{M}_{1.1}$, and X-CNF${ }_{4}-$ $\mathrm{M}_{2.2}$ with varying the matrix content are compared in Fig. 2c-e. Fig. 2c shows CNF bound by the matrix forming an interconnected pore structure with single and bundled fibers emerging from and embedded in the matrix. $\mathrm{X}-\mathrm{CNF}_{4}-\mathrm{M}_{1.1}$ and $\mathrm{X}-\mathrm{CNF}_{4}-\mathrm{M}_{2.2}$ (Fig. 2d and e) show flat and layered structures and resemble self-assembly behaviour similar to that of pure gelatin, as previously found in literature. ${ }^{13,25}$ As the matrix content increased, smoother and thicker wall structures and fewer pores were observed, which was not considered favorable for the pore sizes required for cartilage applications.

When comparing the scaffolds with different initial suspension concentrations, $\mathrm{X}-\mathrm{CNF}_{4}-\mathrm{M}_{0.4}$ showed a pore structure which is relatively homogeneous and interconnected, whereas $\mathrm{X}-\mathrm{CNF}_{5}-\mathrm{M}_{0.5}$ and $\mathrm{X}-\mathrm{CNF}_{6}-\mathrm{M}_{0.6}$ showed more of a layered structure than a fibrillar structure and formed denser structures with fewer pores and cellulose nanofibers coated with matrix compared to $\mathrm{X}-\mathrm{CNF}_{4}-\mathrm{M}_{0.4}$ (images given in ESI, S1 $\dagger$ ). Earlier studies also have demonstrated the controlling the cell size and foam density by changing the suspension concentration. ${ }^{26,27}$

The pore sizes measured from SEM for all developed scaffolds are summarized in Table 1 . In all cases the standard deviations are high and pore sizes in the range of $20 \mu \mathrm{m}$ to 200 $\mu \mathrm{m}$ were observed. The highest average pore size $(100-200 \mu \mathrm{m})$ was with the uncrosslinked system and the pore size decreased with crosslinking (20-120 $\mu \mathrm{m})$, increased matrix content (20-75 $\mu \mathrm{m})$ and increased suspension concentration (40-115 $\mu \mathrm{m})$.

The $\mathrm{X}-\mathrm{CNF}_{4}-\mathrm{M}_{0.4}$ system was considered optimal for the cartilage tissue engineering based on pore structure, homogeneity of pores and average pore sizes. When examined using high-resolution microscopy (Fig. 2f), X-CNF $-\mathrm{M}_{0.4}$ showed a highly entangled network of CNF on the pore walls. These fibrous nanostructures and the pore wall roughness is expected to aid cell fixation and extracellular matrix (ECM) development after implantation because the rougher surface improves vascularization, diffusion rates to and from the scaffold for oxygen/nutrients supply and removal of waste. ${ }^{23,28}$ (For this system, the nanoscaled pores measured from micromeritics porosity analyser were in the range of 12-14 $\mathrm{nm}$ ).

All of the materials were highly porous (>93\%) and had low densities, shown in Table 1, and which agree with literature values. ${ }^{27}$ The crosslinking as well as the increase in matrix content had limited influence on porosity. These samples had a similar density irrespective of the matrix content (samples 1$5)$, most likely due to the higher bulk density of cellulose (1.54 g $\mathrm{cm}^{-3}$ ) as cellulose is the main component in all of the samples. When the concentration of the freeze-drying suspension increased (samples 3, 6 and 7) while keeping the fibers to matrix ratio constant $(10: 1)$, the density increased from 0.066 to 0.093 $\mathrm{g} \mathrm{cm}^{-3}$ due to denser packing of fiber network and as expected led to a decrease in porosity. ${ }^{26,27}$

Fig. 3a-c shows cross-sectional images of the porous scaffold for the $\mathrm{X}-\mathrm{CNF}_{4}-\mathrm{M}_{0.4}$ system obtained using X-ray tomography. It can be observed that the scaffold has high porosity (confirming the porosity data in Table 1) as well as pores are uniformly distributed in the horizontal as well as vertical sections of the scans. Furthermore, X-ray tomography confirmed the interconnectivity of the pores as well as the fibrillar structure of the scaffolds.

The 3D interconnectivity of pores (Fig. 3d) throughout the scaffold, the high degree of porosity, the hierarchical pore structure with micron sized pores in the bulk and the nanoscaled pores on the walls of scaffolds were considered optimal for the cartilage tissue engineering. ${ }^{26}$

\subsection{Moisture uptake}

As $70-85 \%$ of the weight of natural cartilaginous tissues is water, ${ }^{\mathbf{1}}$ it is important to understand the water uptake by the scaffolds. The PBS uptake values of the submerged samples were in the range of $1000-1677 \%$, depending on the composition. The results of the moisture uptake measurements are 

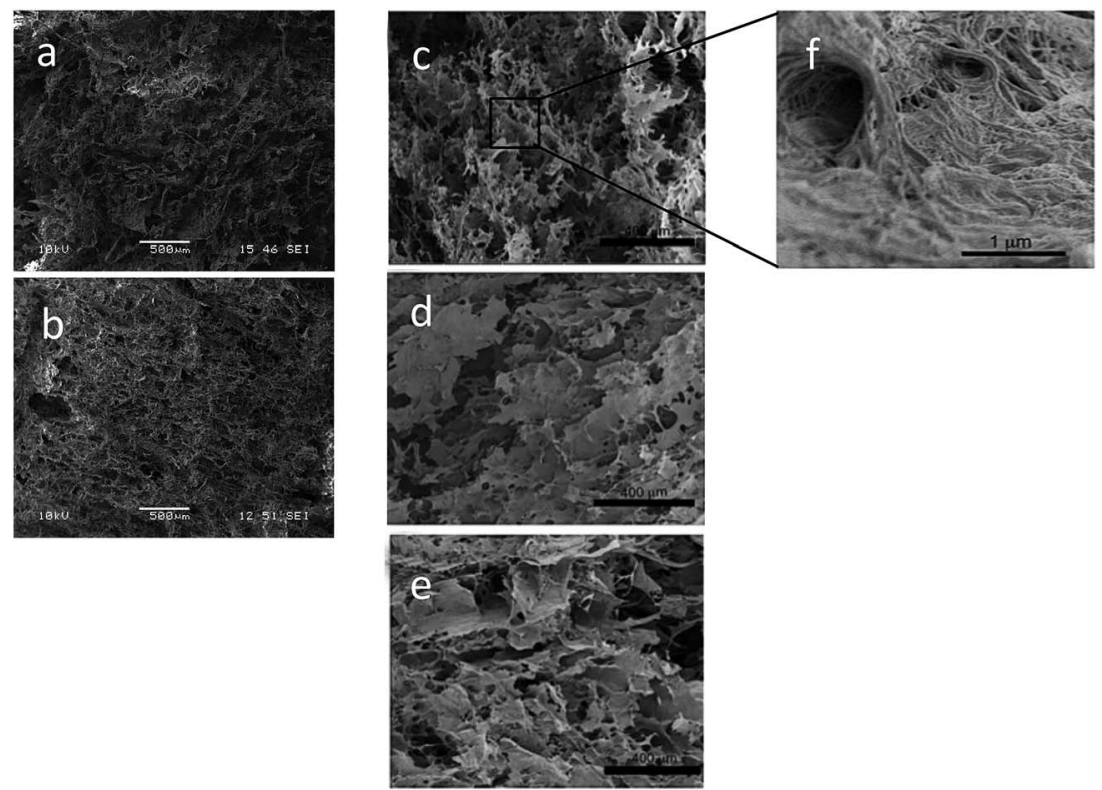

Fig. 2 SEM images comparing the effect of crosslinking on the morphology of scaffolds: (a) $C_{N} F_{4}-M_{0.4}$ and (b) $X-C N F_{4}-M_{0.4}$; overview SEM

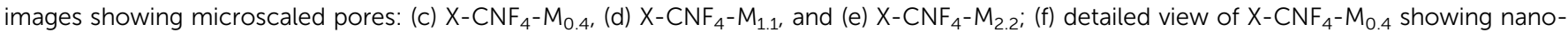
scaled structures on pore walls.
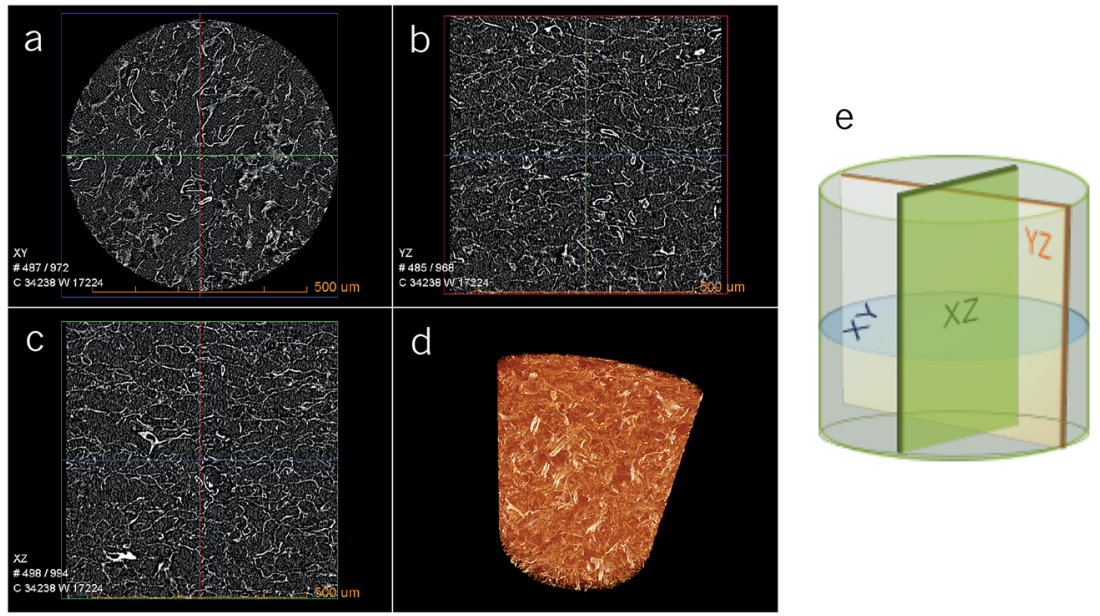

Fig. 3 X-ray tomography showing cross-sectional images for $X-C_{-N} F_{4}-M_{0.4}$ scaffold at different angles: (a) $X Y$, (b) $Y Z$ and (c) $X Z$; (d) $3 D$ reconstruction of the pore structure of the nanocomposite scaffold showing homogeneity of the pores; (e) the schematic representation of the cross-sectioning for imaging.

shown in Fig. 4. The initial moisture uptake was instantaneous (30 s) and remained constant after 3 days in PBS and the materials showed hydrogel behaviour.

It can be inferred that the open pore structure of the materials (shown in SEM) plays a role in the fast and high moisture uptake and moisture susceptibility in the scaffolds. It was found that the amount of matrix does not significantly affect the uptake, as X-CNF $4-\mathrm{M}_{0.4}, \mathrm{X}-\mathrm{CNF}_{4}-\mathrm{M}_{1.1}$ and $\mathrm{X}-\mathrm{CNF}_{4}-\mathrm{M}_{2.2}$ showed similar water uptake. This indicates that a significant proportion of the water uptake is due to the porous structure of the scaffolds. A higher CNF concentration in the suspension resulted in a decrease in the uptake of the resultant scaffolds due to a tighter, denser network with smaller pores, as expected. However, it was noted that porous CNF scaffolds without matrix $\left(\mathrm{CNF}_{4}\right)$ absorbed PBS quickly and disaggregated easily when manipulated since there is no matrix to bind the fibers together. The uncrosslinked samples also proved to be unstable after 3 days in PBS, while the crosslinked samples remained stable in moist conditions during the whole duration of the experiment.

The water uptake by the same scaffolds was monitored in 95\% RH conditions (without immersion) and the maximum uptake was $30 \%$ of its original weight, even after 15 days (shown by Fig. 4 in the inset). This shows that the adsorption due to the scaffold swelling is negligible. No difference has been observed 


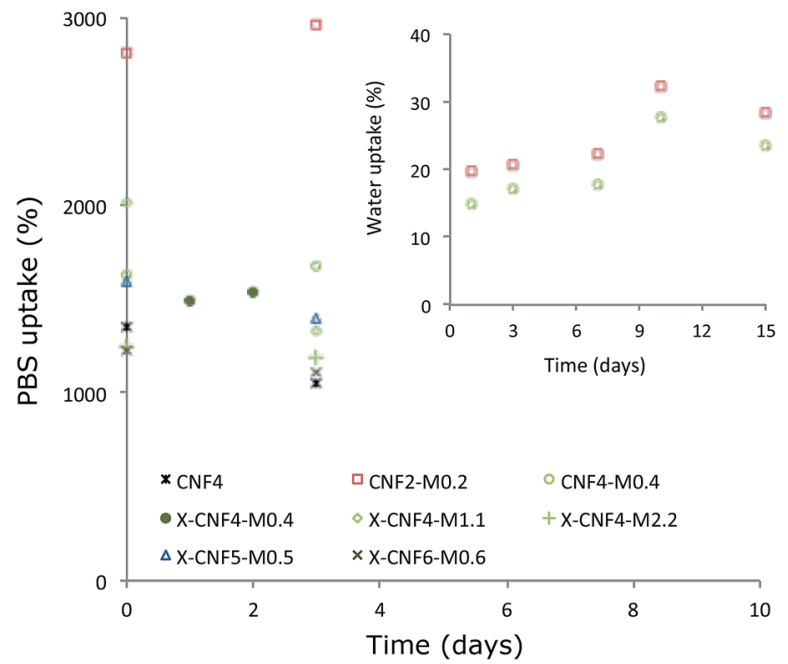

Fig. 4 Effect of fibers/matrix ratio, initial concentration and crosslinking on the PBS and water uptake by the scaffolds.

regardless of whether the samples are crosslinked or not, and the total uptake showed a tendency to decrease as the initial concentration increased, which is in correlation with the density of the material.

\subsection{Mechanical properties}

Compression is the preferred mode of mechanical testing for cartilage materials because the role of natural cartilage is to bear loads in compression ${ }^{1,13}$ (see ESI for the representative load displacement curves, S2 $\dagger$ ). In dry conditions and at $37{ }^{\circ} \mathrm{C}$ (Fig. 5a), the compression modulus was in the range 1-3 MPa which agree with earlier reports of anisotropic CNF based foams. ${ }^{26,27}$ No clear trend can be observed when varying the total concentration or the $\mathrm{CNF} /$ matrix ratio and the values do not follow the density as reported in some earlier literature, ${ }^{26}$ most likely due to the crosslinking effect which is not considered in density calculation. Nonetheless, the presence of the matrix enhanced the mechanical properties in dry conditions when compared to CNF alone. However, high standard deviations were observed and may be due to the wide distribution of the pore sizes.

The aggregate compression modulus of articular cartilage is reported to be around $0.9 \mathrm{MPa}$ by Martin et al. ${ }^{29}$ and 0.5-0.1 MPa by Guilak et al. ${ }^{30}$ Also, a wide range of values varying between $0.1 \mathrm{MPa}$ and $2 \mathrm{MPa}$ are reported as compression moduli for healthy cartilage ${ }^{31-34}$ depending on the source and testing conditions. The values of compression moduli of the current scaffolds are slightly higher than that of natural cartilage, but it may be noted that the moisture content in natural cartilage is greater.

The performance of the scaffolds was evaluated in simulated body conditions (PBS medium and $37{ }^{\circ} \mathrm{C}$ ) to understand the effect of compression rate, crosslinking, fibers/matrix ratio and initial suspension concentration; (see Fig. 5b-e). The compression modulus showed a clear tendency to increase as the compression rate increased from 100 to $400 \mu \mathrm{m} \mathrm{min}{ }^{-1}$
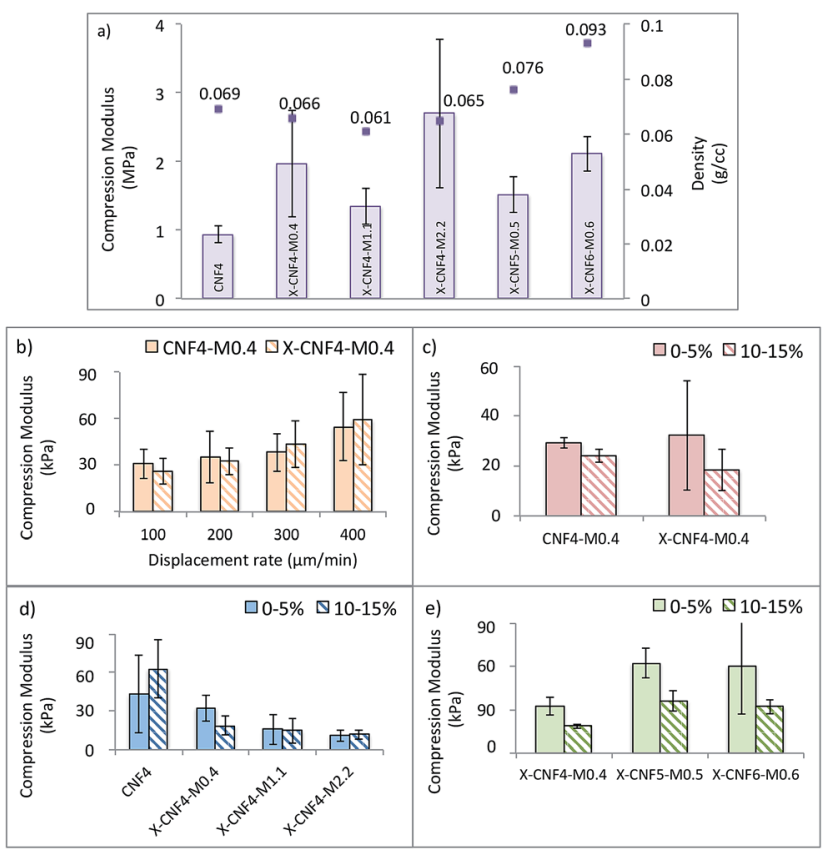

Fig. 5 (a) Average compression moduli of scaffolds in room conditions; graphs showing the (b) effect of compression rate, (c) effect of crosslinking, (d) effect of fibers/matrix ratio and (e) effect of suspension concentration on the compression modulus of the scaffolds tested in simulated body conditions.

(Fig. 5b), a sign of the viscoelasticity of the scaffolds. In dry conditions, the scaffolds exhibited an elastoplastic behaviour, but when submerged in PBS, viscoelastic behaviour was evident. The viscoelastic behaviour in PBS medium is partly due to the swelling of the matrix phase as well as fluid flow through the pores of the scaffolds during compression. This tendency was reported for natural cartilage tissues when tested in compression mode and therefore considered favorable for load bearing by the scaffolds. ${ }^{21}$

The influence of crosslinking on compression modulus was evaluated for $\mathrm{CNF}_{4}-\mathrm{M}_{0.4}$ and X-CNF${ }_{4}-\mathrm{M}_{0.4}$, tested in PBS at 400 $\mu \mathrm{m} \mathrm{min}^{-1}$ (Fig. 5 c) in the strain region of $0-5 \%$ and $10-15 \%$. No significant improvement on the mechanical properties was achieved by crosslinking and the compression modulus was around $30 \mathrm{kPa}$. The low amount of matrix material available for crosslinking as well as the similar density and porosity observed for these scaffolds explains this trend.

The compression moduli of $\mathrm{CNF}_{4}, \mathrm{X}-\mathrm{CNF}_{4}-\mathrm{M}_{0.4}, \mathrm{X}-\mathrm{CNF}_{4}-\mathrm{M}_{1.1}$ and $\mathrm{X}-\mathrm{CNF}_{4}-\mathrm{M}_{2.2}$ in the strain region of $0-5 \%$ and $10-15 \%$ are given in Fig. $5 \mathrm{~d}$. The values decreased from $62 \mathrm{kPa}$ for pure CNF to $10 \mathrm{kPa}$ for nanocomposites with the highest matrix concentration $\left(\mathrm{X}-\mathrm{CNF}_{4}-\mathrm{M}_{2.2}\right)$. The results show that the scaffolds become weaker in wet conditions when the matrix content increases. One possible reason is that gelatin and chitosan are mechanically weaker in wet conditions than the CNF network. Nevertheless, a minimum concentration of matrix phase was necessary to bind the fibers together and ensure the stability of the scaffold in wet medium. Furthermore, $\mathrm{CNF}_{4}$ collapsed completely during compression tests in submersion mode after $15 \%$ strain, while scaffolds with matrix phase showed better 
mechanical and dimensional stability in spite of the lower compression moduli. Fig. 5e shows scaffolds prepared by

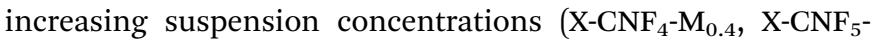
$\mathrm{M}_{0.5}$ and $\left.\mathrm{X}-\mathrm{CNF}_{6}-\mathrm{M}_{0.6}\right)$ but the same ratio of fibers/matrix $(10: 1)$. The compression modulus increased from 18 to 60 $\mathrm{kPa}$ as the concentration increased from 2.2 to 5.5 but stabilized thereafter. The increase in density of the scaffolds did not affect the mechanical properties significantly, especially in PBS medium.

In general, it can be seen that the compression modulus in wet conditions is lower $(10-60 \mathrm{kPa})$ than in dry conditions (1-3 $\mathrm{MPa})$. The decreased mechanical properties in wet conditions were expected due to the swelling and plasticisation of the scaffold pore walls with water. The hydrophilicity of the nanocellulose, gelatin and chitosan as well as the high water-binding capability of gelatin significantly impacts the performance in aqueous medium. The compression moduli of the scaffolds with CNF as reinforcement in the chitosan/gelatin matrix is however significantly higher than those reported for the chitosan/gelatin blend, ${ }^{18}$ indicating that CNF acts as reinforcements in the porous scaffold.

The values presented here are lower than reported for natural cartilage tested in wet conditions. It may be noted that the current compression studies are performed submerged in PBS and at $37{ }^{\circ} \mathrm{C}$ which can weaken the scaffold in comparison to conditioned natural cartilage tested at $37^{\circ} \mathrm{C}$. More importantly, it was demonstrated earlier that in in vivo conditions, chondrocytes sense the mechanical properties of the substrate and soft scaffolds (4 kPa) facilitate chondrogenesis where as stiffer scaffolds $\left(\geq 40 \mathrm{kPa}\right.$ ) are shown to favor bone regeneration. ${ }^{35,36}$

In the current study, $\mathrm{X}-\mathrm{CNF}_{4}-\mathrm{M}_{0.4}$ scaffolds have shown favorable mechanical properties $(18-32 \mathrm{kPa})$ for chondrogenesis, stability in moist conditions and favorable pore sizes and pore wall morphology for cell adhesion. Therefore, these scaffolds are expected to develop extracellular matrix (ECM) and regenerate cartilage with the right mechanical properties after implantation. The evaluation of the scaffold after ECM development will be required to understand the performance of the scaffold in in vivo conditions and may be addressed in future.

\subsection{Biodegradability}

In vitro degradation tests of the scaffolds were investigated up to 28 days and the results are shown in Fig. 6 . The scaffold, which contained only $\mathrm{CNF}_{4}$ (used as control), displayed significant morphological changes during the degradation time. In agreement with the observations during water uptake studies, these scaffolds did not have dimensional stability in order to bind the fibers together due to lack of matrix content. The scaffold comprising $\mathrm{X}-\mathrm{CNF}_{4}-\mathrm{M}_{2.2}$ showed around $27 \%$ decrease in weight, while the scaffold comprising $\mathrm{X}-\mathrm{CNF}_{4}-\mathrm{M}_{0.4}$ and $\mathrm{X}-\mathrm{CNF}_{6}-$ $\mathrm{M}_{0.6}$ demonstrated $1.5 \%$ weight loss after up to 4 weeks. Therefore, the greatest weight loss was obtained for the scaffold containing the higher amount of matrix.

An optimal rate of degradation crucial for cartilage regeneration is one which balances stable $3 \mathrm{D}$ structures that provide support with gradual development of ECM. ${ }^{37}$

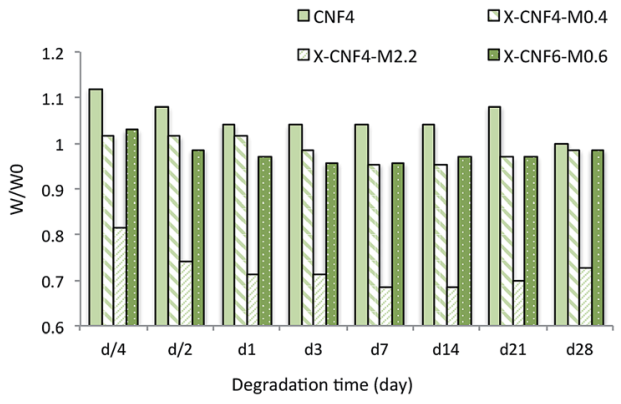

Fig. 6 Biodegradation of scaffolds in PBS medium at $37^{\circ} \mathrm{C}$

The short-term biodegradation studies showed that the rate of degradation of nanofibrous scaffolds could be controlled by the fibers/matrix ratio. The slow biodegradation tendency of biologically stable scaffolds in the current study, which provides an enduring support for the patient while also favoring ECM formation, can be considered beneficial. However, further longterm investigation needs to be done to evaluate ECM development in correlation with biodegradation of the scaffold to ensure the potential of the produced scaffold.

\subsection{Cytocompatibility studies}

Cellulose nanofibers. The cytocompatibility of CNF was evaluated using a direct contact testing system and the results are shown in Fig. 7a and b. The L929 cell line was chosen due to
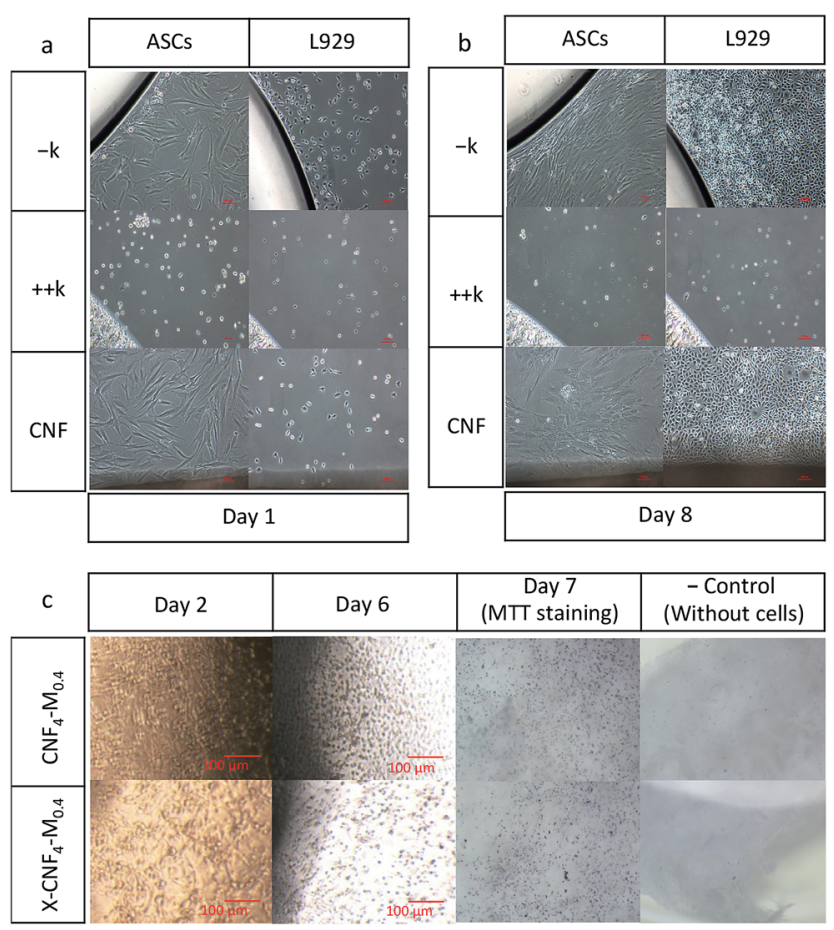

Fig. 7 Cytocompatibility of the cellulose nanofibers towards adipose derived stem cells (ASCs) and L929 cell line, (a) at day 1 and (b) day 8; cytocompatibility results for $\mathrm{CNF}_{4}-\mathrm{M}_{0.4}$ towards chondrocytes before and after crosslinking (c). 
ISO 10993 recommendations and (ASCs) were chosen due to their fibroblast morphology, sensibility and their role as a chondrocyte precursor. In addition to CNF films, noncytotoxic $(-\mathrm{K})$ and cytotoxic $(++\mathrm{K})$ controls were also used to measure cytotoxicity effects. The cells in the presence of negative control $(-\mathrm{K})$ exhibited efficient proliferation between day 1 and 8 , whereas positive control $(++\mathrm{K})$ showed no cell attachment or growth. This short-term cytocompatibility test results indicate that CNF support cell growth and can have potential in biomedical scaffold fabrication. We have also recently reported these CNF are cytocompatible according to current ISO criteria, with non-inflammatory and non-immunogenic properties. ${ }^{20}$ Higher concentrations were found to be tolerogenic to the immune system, a characteristic very desirable for implantable biomaterials, which justifies the use of wood-based CNF in the current application.

3D porous scaffold. When cells seeded on biomaterial surface, they attached to the scaffold. However, the cells retained the circular morphology up to day 6 , but showed no sign of zone of inhibition or reduced growth, as shown in Fig. 7c. At day 7, the samples were stained with MTT in order to highlight the live cells, as the enzymes in live cells catalyze the reaction, resulting in a purple colored product. All seeded samples were compared with samples without cells, as a negative control. The morphology of the cells was round, which indicates that the cells are being encapsulated in the scaffold, as also seen in some other hydrogels which are routinely and effectively used in clinical practice. ${ }^{38}$ As shown in Fig. 7c, the chondrocytes in the scaffolds remained viable after 7 days. Therefore, the material is regarded as non-cytotoxic and is suitable for further evaluation.

\section{Conclusions}

Nanocomposites of cellulose nanofibers bound in a gelatin and chitosan matrix were prepared via freeze-drying and crosslinked using genipin to obtain highly porous ( $\approx 95 \%$ porosity) $3 \mathrm{D}$ scaffolds with optimal pore size, porosity, pore interconnectivity as well as mechanical performance, moisture stability and cell interactions. The freeze-drying route resulted in isotropic scaffolds and the pore structure was most homogenous for nanocomposites with a low amount of crosslinked matrix that acted as binding phase and dimensional stabilizer in moist conditions. The compression moduli of the optimal scaffolds $\left(\mathrm{X}-\mathrm{CNF}_{4}-\mathrm{M}_{0.4}\right)$ in dry conditions, at $37{ }^{\circ} \mathrm{C}$, were around $1 \mathrm{MPa}$ and considered comparable to natural cartilaginous tissue. These scaffolds showed viscoelasticity when immersed in PBS, similar to that of natural cartilage, but lower compression modulus (18-32 kPa) which was considered favorable for soft tissue regeneration. We have successfully tailored fully bio-based scaffolds with a high degree of bulk porosity, hierarchical pore structure, nanoscaled roughness and fibrillar structure of the pore walls combined with good mechanical properties and cytocompatibility with high potential for cartilage regeneration. Furthermore, the possibility to develop ECM and trap moisture in the interconnected pore structure is expected to bring the mechanical properties closer to those of natural cartilage after implantation.

\section{Acknowledgements}

The authors gratefully acknowledge VINNOVA for financial support under the MNT-ERANET project n-POSSCOG (201102071). The authors would also like to thank Iftekhar Uddin Bhuiyan and Stephen Hall for help with high-resolution SEM images and X-ray tomography, respectively. Thushara Thomas is acknowledged for the help with water sorption studies.

\section{Notes and references}

1 J. M. Mansour, Kinesiology: the mechanics and pathomechanics of human movement, 2003, pp. 66-79.

2 E. B. Hunziker, Osteoarthritis Cartilage, 2002, 10, 432-463.

3 J. K. Francis Suh and H. W. T. Matthew, Biomaterials, 2000, 21, 2589-2598.

4 S. W. Whu, K. Hung, K. Hsieh, C. Chen, C. Tsai and S. Hsu, Mater. Sci. Eng., C, 2013, 33, 2855-2863.

5 S. Bhat, A. Tripathi and A. Kumar, J. R. Soc., Interface, 2011, 8, 540-554.

6 N. S. Sambudi, M. Sathyamurthy, G. M. Lee and S. B. Park, Compos. Sci. Technol., 2015, 106, 76-84.

7 Y. Zhao, S. Gao, S. Zhao, Y. Li, L. Cheng, J. Li and Y. Yin, Mater. Sci. Eng., C, 2012, 32, 2153-2162.

8 A. P. Mathew, K. Oksman, D. Pierron and M. Harmand, Macromol. Biosci., 2013, 13, 289-298.

9 A. P. Mathew, K. Oksman, D. Pierron and M. Harmad, Cellulose, 2012, 19, 139-150.

10 K. Tomihata, K. Burczak, K. Shiraki and Y. Ikada, in Polymers of Biological and Biomedical Significance, nonymous, ACS, 1993, pp. 275-286.

11 J. Hodde, Tissue Eng., 2002, 8, 295-308.

12 Z. Huang, Y. Z. Zhang, M. Kotaki and S. Ramakrishna, Compos. Sci. Technol., 2003, 63, 2223-2253.

13 M. Sarem, F. Moztarzadeh and M. Mozafari, Carbohydr. Polym., 2013, 93, 635-643.

14 N. Naseri, C. Algan, V. Jacobs, M. John, K. Oksman and A. P. Mathew, Carbohydr. Polym., 2014, 109, 7-15.

15 N. Naseri, A. P. Mathew, L. Girandon, M. Fröhlich and K. Oksman, Cellulose, 2015, 22, 521-534.

16 D. W. Hutmacher, Biomaterials, 2000, 21, 2529-2543.

17 V. Chiono, E. Pulieri, G. Vozzi, G. Ciardelli, A. Ahluwalia and P. Giusti, J. Mater. Sci.: Mater. Med., 2008, 19, 889-898.

18 A. Arora, A. Kothari and D. S. Katti, J. Mech. Behav. Biomed. Mater., 2015, 51, 169-183.

19 A. P. Mathew, K. Oksman, Z. Karim, P. Liu, S. A. Khan and N. Naseri, Ind. Crops Prod., 2014, 58, 212-219.

20 M. Colic, D. Mihajlovic, A. Mathew, N. Naseri and V. Kokol, Cellulose, 2014, 22, 763-778.

21 H. N. Chia and M. L. Hull, J. Orthop. Res., 2008, 26, 951-956. 22 H. Sehaqui, Q. Zhou, O. Ikkala and L. A. Berglund, Biomacromolecules, 2011, 12, 3638-3644.

23 A. Thorvaldsson, H. Stenhamre, P. Gatenholm and P. Walkenström, Biomacromolecules, 2008, 9, 1044-1049.

24 T. Telemeco, C. Ayres, G. Bowlin, G. Wnek, E. Boland, N. Cohen, C. Baumgarten, J. Mathews and D. Simpson, Acta Biomater., 2005, 1, 377-385. 
25 J. S. Mao, L. G. Zhao, Y. J. Yin and K. de Yao, Biomaterials, 2003, 24, 1067-1074.

26 H. Sehaqui, M. Salajková, Q. Zhou and L. A. Berglund, Soft Matter, 2010, 6, 1824-1832.

27 H. Sehaqui, Q. Zhou and L. A. Berglund, Compos. Sci. Technol., 2011, 71, 1593-1599.

28 H. Cheung, K. Lau, T. Lu and D. Hui, Composites, Part B, 2007, 38, 291-300.

29 R. B. Martin, D. B. Burr and N. A. Sharkey, in Skeletal Tissue Mechanics, ed. R. B. Martin, D. B. Burr and N. A. Sharkey, Springer, New York, 1998, pp. 278-289.

30 F. Guilak, W. R. Jones, H. P. Ting-Beall and G. M. Lee, Osteoarthritis and Cartilage, 1999, 7, 59-70.

31 J. Jurvelin, I. Kiviranta, J. Arokoski, M. Tammi and H. Helminen, Eng. Med., 1987, 16, 15-22.
32 M. Laasanen, J. Töyräs, R. Korhonen, J. Rieppo, S. Saarakkala, M. Nieminen, J. Hirvonen and J. Jurvelin, Biorheology, 2003, 40, 133-140.

33 A. J. Almarza and K. A. Athanasiou, Ann. Biomed. Eng., 2004, 32, 2-17.

34 K. L. Spiller, J. L. Holloway, M. E. Gribb and A. M. Lowman, J. Tissue Eng. Regener. Med., 2011, 5, 636-647.

35 E. Schuh, J. Kramer, J. Rohwedel, H. Notbohm, R. Müller, T. Gutsmann and N. Rotter, Tissue Eng., Part A, 2010, 16, 1281-1290.

36 W. Zhong, Y. Li, L. Li, W. Zhang, S. Wang and X. Zheng, J. Mol. Histol., 2013, 44, 587-595.

37 J. Raghunath, J. Rollo, K. M. Sales, P. E. Butler and A. M. Seifalian, Biotechnol. Appl. Biochem., 2007, 46, 73-84.

38 L. Flynn, G. D. Prestwich, J. L. Semple and K. A. Woodhouse, Biomaterials, 2007, 28, 3834-3842. 